\title{
A fractional-step finite-element method for the Navier- Stokes equations applied to magma-chamber withdrawal
}

\author{
Arnau Folch ${ }^{\mathrm{a}, *}$, Mariano Vázquez ${ }^{\mathrm{b}}$, Ramon Codina ${ }^{\mathrm{b}}$, Joan Martí ${ }^{\mathrm{a}}$ \\ anstitute of Earth Sciences 'Jaume Almera', CSIC, Lluís Solé Sabarís s/n, 08028 Barcelona, Spain \\ ${ }^{\mathrm{b}}$ International Center for Numerical Methods in Engineering, Universitat Politècnica de Catalunya, Gran Capità s/n, 08034 Barcelona, \\ Spain
}

Received 1 December 1997; received in revised form 29 October 1998

\begin{abstract}
We develop an algorithm to solve numerically the Navier-Stokes equations using a finite element method. The algorithm uses a fractional step approach that allows the use of equal interpolation spaces for the pressure and velocity fields and can be used to solve both compressible and incompressible flows. The standard Galerkin method is used to space-discretize the equations in which convective terms are dominant because the equations are reformulated in a characteristics co-moving frame providing thus the required artificial diffusion in a consistent way. The algorithm depends on four different parameters. Depending on their values, a fully implicit, a semi-implicit or a fully explicit solution can be obtained. The proposed algorithm may be useful in solving a wide spectrum of problems in geology, engineering and geosciences. Several flows frequently encountered in practical applications such as incompressible, slightly compressible or perfect gas are taken into account. As an example, we use it to model the withdrawal of magma from shallow chambers during explosive volcanic eruptions. Our model constitutes a first attempt to characterize the temporal evolution of the most relevant physical parameters during such a process. (C) 1999 Elsevier Science Ltd. All rights reserved.
\end{abstract}

\section{Code available at http.//www.iamg.org/cGEditor/index.htm}

Keywords: Navier-Stokes equations; Finite element method; Fractional step method; Explosive volcanic eruptions; Magma chamber withdrawal

\section{Introduction}

Magmas are silicate melts containing dissolved volatile species whose solubility depends mainly on pressure. At a given temperature and volatile content, there is a critical pressure $P_{\mathrm{c}}$ at which the exsolution of a gas species begins due to oversaturation of the melt. Below this critical pressure, corresponding to a critical

\footnotetext{
* Corresponding author. Tel.: +34-3-409-5410; fax: +34-3411-0012; e-mail: afolch@ija.csic.es
}

depth or exsolution level, the magma behaves approximately as incompressible. In contrast, above the exsolution level, where the pressure is lower than $P_{\mathrm{c}}$, the magma becomes a compressible two-phase mixture of liquid and dispersed gas bubbles (Sparks, 1978; Papale, 1996). Silicic magma is frequently stored in shallow chambers located a few kilometers below the Earth's surface (Smith, 1979). When pressure inside the chamber is greater than lithostatic, the chamber is said to be overpressured. When the overpressure exceeds the tensile strength of surrounding rocks, a volcanic eruption may be triggered through the propagation of 
fractures to the surface. The increase in magma pressure necessary to trigger an eruption can be produced either by the addition of new magma into the chamber (Blake, 1981) or by the exsolution of volatiles associated with cooling and crystallization of the magma (Blake, 1984; Tait et al., 1989). In the second situation, the exsolution level must be located inside the chamber.

So far, available numerical simulations of the withdrawal process consider the chamber as an open system; that is, the withdrawal process is driven only by pressure forces associated with magma replenishment and always assume the magma to be incompressible (Spera, 1984; Spera et al., 1986; Trial et al., 1992). Folch et al. (1998) make a first attempt to characterize the temporal evolution of the physical properties of the magma inside the chamber during a volcanic eruption from a closed system, where the eruption is triggered by volatile oversaturation. In such a situation, magma should be treated as an incompressible Newtonian flow below the exsolution level and as a compressible, homogeneous two-phase flow above it. Therefore, an algorithm able to deal simultaneously and well with compressible and incompressible flows is required.

In this paper, the numerical method employed in Folch et al. (1998) is described in detail and extended to other situations of interest sent an algorithm that, using (FEM), numerically solves

equations. It uses a fractional-step method which fllows the use of equal interpolation spaces for the pressure and the velocity fie ds and produces a stabilizing effect on the pressure, thus eliminating the need for a special interpolation when the incompressible limit is

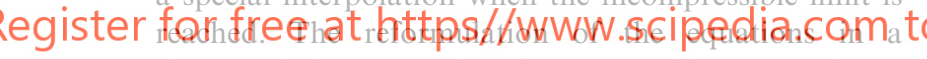
characteristics co-moving frame provides a consistent artificial diffusion when the spatial discretization is done. This diffusion, similar to that of the SUPG methods, can handle the purely numerical oscillations which usually appear when the Galerkin method is applied to equations with dominant convective terms.

\section{The algorithm}

\subsection{Navier-Stokes equations}

The equations that model the behavior of a Newtonian fluid are the Navier-Stokes equations, derived from general conservation principles of mass, momentum and energy. A simplified version of these equations can be written as the continuity equation (e.g. Batchelor, 1967; Faber, 1995),

$\frac{\partial \rho}{\partial t}+\frac{\partial U_{i}}{\partial x_{i}}=0$,

the momentum equation, $\frac{\partial U_{j}}{\partial t}+\frac{\partial\left(u_{i} U_{j}\right)}{\partial x_{i}}+\frac{\partial}{\partial x_{i}}\left(\delta_{i j} p-\tau_{i j}\right)+\rho g_{j}=0$,

and the heat equation,

$\frac{\partial T}{\partial t}+u_{i} \frac{\partial T}{\partial x_{i}}-\frac{1}{c_{\mathrm{v}} \rho} \frac{\partial}{\partial x_{i}}\left(k \frac{\partial T}{\partial x_{i}}\right)-\frac{1}{c_{\mathrm{v}} \rho} \sigma_{i j} \frac{\partial u_{i}}{\partial x_{j}}=0$.

Table 1

List of symbols used in paper. We also employ $f^{n+\vartheta} \equiv \vartheta f^{n+1}+(1-\vartheta) f^{n}$ and $\Delta f^{n} \equiv f^{n+1}-f^{n}$ for any function $f$. Superscript denotes time-step level. Bold vectors stand for vectors of nodal unknowns, and $\overrightarrow{\mathbf{F}}_{\mathrm{i}}$ denotes vector which is known at moment of solving particular equation

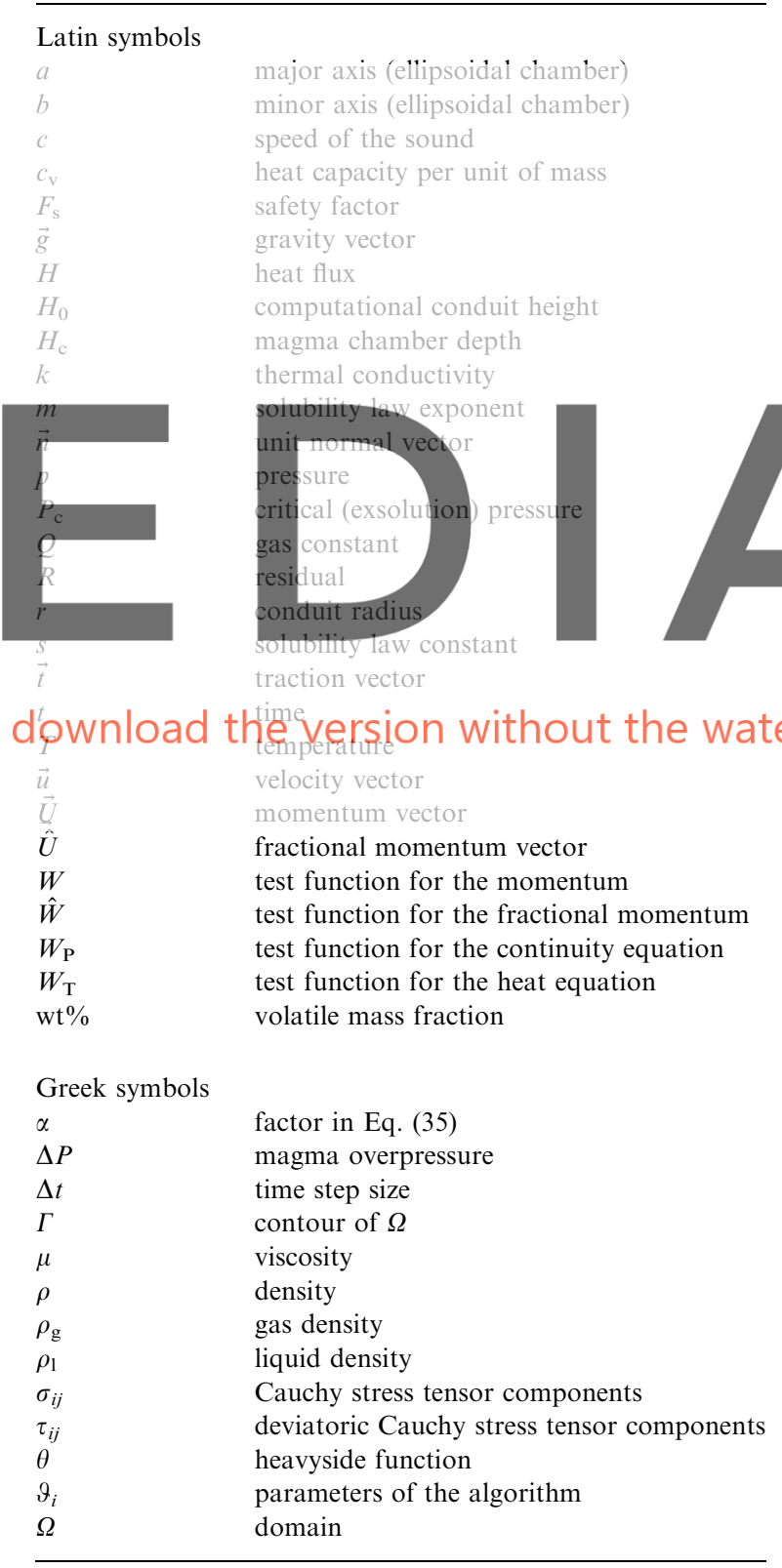


In these equations (see Table 1 for notation) $t$ is time, $\rho$ is density, $U_{i}$ and $u_{i}$ are the $i$ th component of the momentum and velocity vectors, respectively (i.e., $\left.U_{i}=\rho u_{i}\right), p$ is the pressure, $g_{i}$ is the $i$ th component of the gravity vector, $\sigma_{i j}$ and $\tau_{i j}$ are the components of the Cauchy stress tensor and its deviatoric (i.e. $\tau_{i j}=$ $\left.\sigma_{i j}-p \delta_{i j}\right), k$ is the thermal conductivity, $T$ is the temperature and $c_{\mathrm{v}}$ is the heat capacity per unit of mass. In all the equations that appear in this paper, indices run from 1 to 2 or 3 (space dimensions) and repeated indices always imply summation. Assuming the Stokes hypothesis, the deviatoric stress tensor $\tau_{i j}$ is linearly related to velocity gradients by

$\tau_{i j}=\mu\left(\frac{\partial u_{i}}{\partial x_{j}}+\frac{\partial u_{j}}{\partial x_{i}}-\frac{2}{3} \frac{\partial u_{k}}{\partial x_{k}} \delta_{i j}\right)$

where $\mu$ is the viscosity. Adding a state law

$\varphi(\rho, T, p)=0$

it is possible to solve Eqs. (1)-(5) numerically.

2.2. Time discretization

It can be shown that when a scalar equation like:

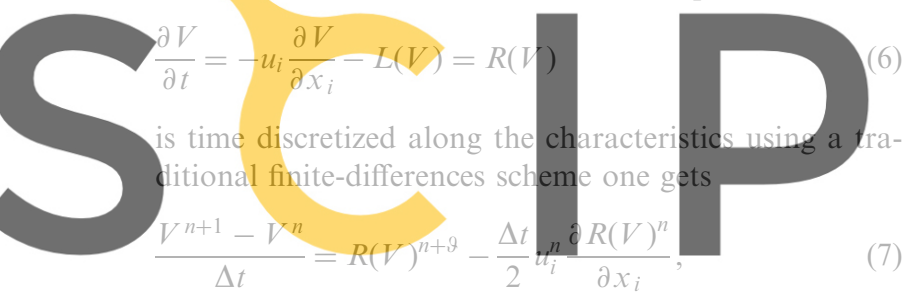

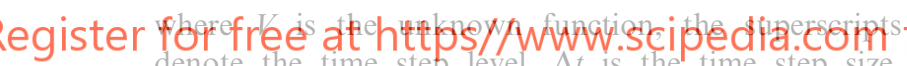
denote the time step level, $\Delta t$ is the time step size, any function $f$ and $R(V)$ is the residual. We will later show how the term that is proportional to $\Delta t$ in Eq. (7) becomes a SUPG-like term when the space discretization is done, i.e. is a term that produces a stabilizing effect in those equations with convective terms by adding numerical diffusion along the streamlines in a consistent manner. We use Eq. (7) to discretize in time both the momentum equation, Eq. (2), and the heat equation, Eq. (3), because these equations contain convective terms that could lead to numerical instabilities. This leads to the time discretized continuity equation:

$\frac{\Delta \rho^{n}}{\Delta t}=-\frac{\partial U_{i}^{n+\vartheta_{1}}}{\partial x_{i}}$

the time discretized momentum equation:

$\frac{\Delta U_{i}^{n}}{\Delta t}=M_{i}^{n+\vartheta_{3}}-\frac{\partial p^{n+\vartheta_{2}}}{\partial x_{i}}-\frac{\Delta t}{2} u_{k}^{n} \frac{\partial R_{i}^{n}}{\partial x_{k}}$,

where $M_{i}$ and the $i$ th component of the residual $R_{i}$ are defined as:
$M_{i} \equiv-\frac{\partial}{\partial x_{j}}\left(\rho u_{i} u_{j}-\tau_{i j}\right)-\rho g_{i}$,

$R_{i} \equiv M_{i}-\frac{\partial p}{\partial x_{i}}$

and the time discretized heat equation:

$\frac{\Delta T^{n}}{\Delta t}=R_{\mathrm{T}}^{n+\vartheta_{4}}-\frac{\Delta t}{2} u_{k}^{n} \frac{\partial R_{\mathrm{T}}^{n}}{\partial x_{k}}$,

where the residual of the heat equation $R_{\mathrm{T}}$ is defined as:

$R_{\mathrm{T}} \equiv-u_{i} \frac{\partial T}{\partial x_{i}}+\frac{1}{c_{\mathrm{v}} \rho} \frac{\partial}{\partial x_{i}}\left(k \frac{\partial T}{\partial x_{i}}\right)+\frac{1}{c_{\mathrm{v}} \rho} \sigma_{i j} \frac{\partial u_{i}}{\partial x_{j}}$.

In these equations $\Lambda f^{n} \equiv f^{n+1}-f^{n}$ for any function $f$ and $\vartheta_{1}, \vartheta_{2}, \vartheta_{3}$ and $\vartheta_{4} \in[0,1]$ are parameters introduced during the discretization. We will later show how, depending on its values, the algorithm becomes explicit, semi-implicit or implicit.

\subsection{Splitting of the momentum equation}

The most significant feature of the numerical scheme proposed here is the splitting of the momentum

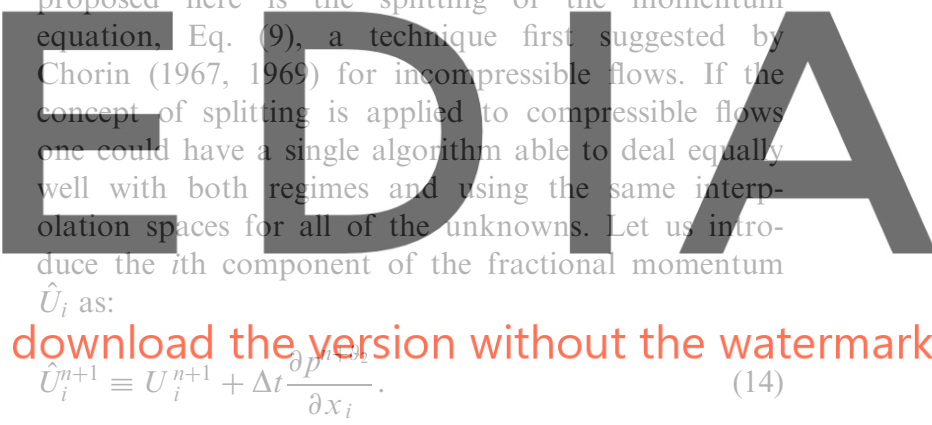

Using Eq. (14), Eqs. (8) and (9) can be rewritten as:

$\frac{\Delta \hat{U}_{i}^{n}}{\Delta t}=M_{i}^{n+\vartheta_{3}}-\frac{\Delta t}{2} u_{k}^{n} \frac{\partial R_{i}^{n}}{\partial x_{k}}$,

$\frac{\Delta \rho^{n}}{\Delta t}=-\frac{\partial}{\partial x_{i}}\left(U_{i}^{n}+\vartheta_{1} \Delta \hat{U}_{i}^{n}-\vartheta_{1} \Delta t \frac{\partial p^{n+\vartheta_{2}}}{\partial x_{i}}\right)$,

$\frac{\Delta U_{i}^{n}}{\Delta t}=\frac{\Delta \hat{U}_{i}^{n}}{\Delta t}-\frac{\partial p^{n+\vartheta_{2}}}{\partial x_{i}}$.

We first compute the fractional momentum using Eq. (15), then the density (or the pressure) using Eq. (16) and finally the momentum by using Eq. (17). A deeper insight into the implicit treatment of $M_{i}$ in Eq. (15) can be achieved by separating its convective and viscous parts, defined, respectively, as:

$M_{\mathrm{c}, i} \equiv-\frac{\partial}{\partial x_{i}}\left(\rho u_{i} u_{j}\right)=-\frac{\partial}{\partial x_{j}}\left(u_{j} U_{i}\right)$ 


$$
\begin{aligned}
\int_{\Omega} W_{\mathrm{P}} \frac{\Delta \rho^{n}}{\Delta t} \mathrm{~d} \Omega & \\
= & -\int_{\Omega} W_{\mathrm{P}} \frac{\partial U_{i}^{n}}{\partial x_{i}} \mathrm{~d} \Omega \\
& +\vartheta_{1} \int_{\Omega} \frac{\partial W_{\mathrm{P}}}{\partial x_{i}}\left(\Delta \hat{U}_{i}^{n}-\Delta t \frac{\partial p^{n+\vartheta_{2}}}{\partial x_{i}}\right) \mathrm{d} \Omega \\
& -\vartheta_{1} \int_{\Gamma} W_{\mathrm{P}} n_{i}\left(\Delta \hat{U}_{i}^{n}-\Delta t \frac{\partial p^{n+\vartheta_{2}}}{\partial x_{i}}\right) \mathrm{d} \Gamma
\end{aligned}
$$

As a boundary condition for the continuity equation we impose that the normal component of Eq. (17) also be verified on the boundary $\Gamma$, a condition equivalent to impose that the normal component of the momentum equation, Eq. (9), be verified on the boundary $\Gamma$. This leads to:

$n_{i}\left(\Delta \hat{U}_{i}^{n}-\Delta t \frac{\partial p^{n+\vartheta_{2}}}{\partial x_{i}}\right)=n_{i} \Delta U_{i}^{n}$

on the part of the boundary $\Gamma_{\mathrm{C}}$ where the test function $W_{\mathrm{P}}$ for the continuity equation does not vanish. On the other hand, the normal component $n_{i} \Delta U_{i}^{n}$ is known on the part of the boundary $\Gamma_{\mathrm{D}}$ where the momentum is given, so that

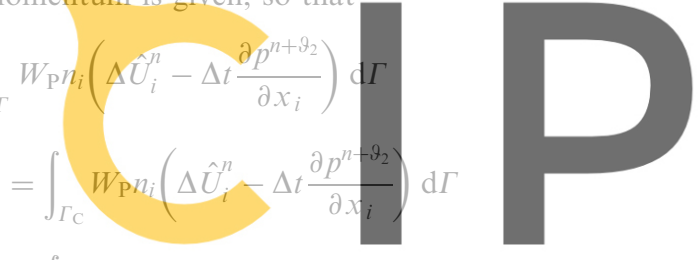

$=\int_{F_{\mathrm{P}}} n_{i} \Delta U_{i}^{n} \mathrm{~d} \Gamma$

(27)

egister for free at https//www.scipedia.com t

The boundary condition introduced by Eq. (27) can then be considered as the normal component of the momentum equations.

\subsubsection{Momentum equation}

For Eq. (17), after weighting by the $i$ th component of the test function for the momentum $W_{i}$ we obtain:

$$
\begin{aligned}
& \int_{\Omega} W_{i} \frac{\Delta U_{i}^{n}}{\Delta t} \mathrm{~d} \Omega \\
& =\int_{\Omega} W_{i} \frac{\Delta \hat{U}_{i}^{n}}{\Delta t} \mathrm{~d} \Omega-\int_{\Omega} W_{i} \frac{\partial p^{n+\vartheta_{2}}}{\partial x_{i}} \mathrm{~d} \Omega .
\end{aligned}
$$

\subsubsection{Heat equation}

Let us now weight Eq. (12) using a test function $W_{\mathrm{T}}$, integrate the diffusion term by parts, set $R_{\mathrm{T}}=0$ on $\Gamma$ and prescribe the conduction heat flux to $H$ on a part of the boundary $\Gamma_{\mathrm{H}}$. For simplicity, we assume an explicit time approximation along the characteristics, i.e. we assume $\vartheta_{4}=0$ in Eq. (12). The result is:

$$
\begin{aligned}
\int_{\Omega} W_{\mathrm{T}} & \frac{\Delta T^{n}}{\Delta t} \mathrm{~d} \Omega \\
= & \int_{\Omega} W_{\mathrm{T}}\left[\left(-u_{i}+\frac{k}{c_{\mathrm{v}} \rho^{2}} \frac{\partial \rho}{\partial x_{i}}\right) \frac{\partial T}{\partial x_{i}}\right]^{n} \mathrm{~d} \Omega \\
& +\int_{\Omega} W_{\mathrm{T}}\left(\frac{1}{c_{\mathrm{V}} \rho} \sigma_{i j} \frac{\partial u_{i}}{\partial x_{j}}\right)^{n} \mathrm{~d} \Omega \\
& -\int_{\Omega} \frac{\partial W_{\mathrm{T}}}{\partial x_{i}}\left(\frac{k}{c_{\mathrm{v}} \rho} \frac{\partial T}{\partial x_{i}}\right)^{n} \mathrm{~d} \Omega \\
& +\frac{\Delta t}{2} \int_{\Omega} \frac{\partial}{\partial x_{k}}\left(u_{k}^{n} W_{\mathrm{T}}\right) R_{\mathrm{T}}^{n} \mathrm{~d} \Omega \\
& +\int_{\Gamma_{\mathrm{H}}} \frac{1}{c_{\mathrm{v}} \rho^{n}} W_{\mathrm{T}} H \mathrm{~d} \Gamma .
\end{aligned}
$$

In Eq. (29), Dirichlet conditions are imposed directly on $\Gamma-\Gamma_{\mathrm{H}}$ and Newman conditions (the heat flux $H$ ) are imposed by computing the boundary integral

$\int_{\Gamma_{\mathrm{H}}} \frac{1}{c_{\mathrm{V}} \rho^{n}} W_{\mathrm{T}} H \mathrm{~d} \Gamma$

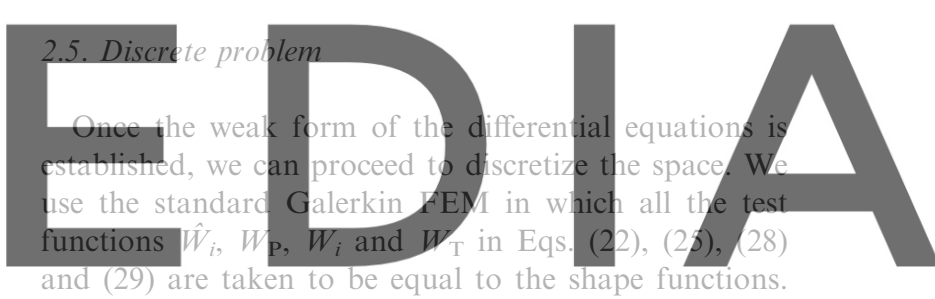

Once the spatial discretization has been performed the

dawnd@ach bheriversionatwithout the watermark

$\mathbf{M} \frac{\Delta \overrightarrow{\hat{U}}^{n}}{\Delta t}=\overrightarrow{\mathbf{F}}_{1}-\mathbf{K} \overrightarrow{\hat{U}}^{n+\theta_{3}}$

$\mathbf{M}_{\alpha} \frac{\Delta \overrightarrow{\mathbf{P}}^{n}}{\Delta t}+\vartheta_{1} \Delta t \mathbf{L} \overrightarrow{\mathbf{P}}^{\mathrm{n}+\vartheta_{2}}+\overrightarrow{\mathbf{F}}_{2}$

$\mathbf{M} \frac{\Delta \overrightarrow{\mathbf{U}}^{\mathrm{n}}}{\Delta t}=\mathbf{M} \frac{\Delta \overrightarrow{\hat{\mathbf{U}}}^{\mathrm{n}}}{\Delta t}-\mathbf{G} \overrightarrow{\mathbf{P}}^{\mathrm{n}+\vartheta_{2}}+\overrightarrow{\mathbf{F}}_{3}$,

$\mathbf{M}_{\mathrm{S}} \frac{\Delta \overrightarrow{\mathbf{T}}^{\mathrm{n}}}{\Delta t}=\overrightarrow{\mathbf{F}}_{4}$,

where $\overrightarrow{\hat{\mathbf{U}}}, \overrightarrow{\mathbf{P}}, \overrightarrow{\mathbf{U}}$ and $\overrightarrow{\mathbf{T}}$ are the vectors of nodal unknowns and $\mathbf{M}, \mathbf{M}_{\mathbf{S}}, \mathbf{K}$ and $\mathbf{G}$ are, respectively, the standard mass matrix for vectorial unknowns, the standard mass matrix for scalar unknowns, the matrix arising from the viscous and convective terms in the fractional momentum equation and the matrix coming from the gradient operator. Here and later we will use $\overrightarrow{\mathbf{F}}$ to denote a vector which is known at the moment of 
solving a particular equation. The matrices $\mathbf{M}_{\alpha}$ and $\mathbf{L}$ in Eq. (32) are defined as:

$\mathbf{M}_{\alpha, \mathrm{ij}} \equiv \int_{\Omega} \alpha N_{i} N_{j} \mathrm{~d} \Omega$

$\mathbf{L}_{\mathrm{ij}} \equiv \int_{\Omega} \frac{\partial N_{i}}{\partial x_{k}} \frac{\partial N_{j}}{\partial x_{k}} \mathrm{~d} \Omega$

where $N_{i}$ is the shape function associated with the $i$ th node of the finite element mesh and $\alpha$ depends on the state law.

\subsection{State law}

In this section we discuss the dependence of the values of $\alpha$ adopted in Eq. (35) on the kind of flow. Although we will apply the algorithm to model the withdrawal of magma from a shallow chamber, let us first consider other flows frequently encountered in engineering and geological applications such as incompressible flow,

$\rho$ constant

slightly compressible flow,

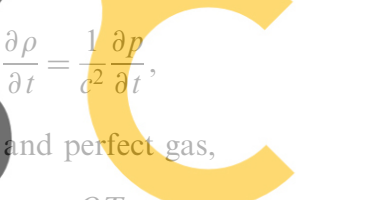

$p=\rho Q T$
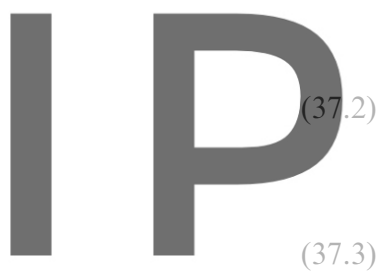

(37.3)

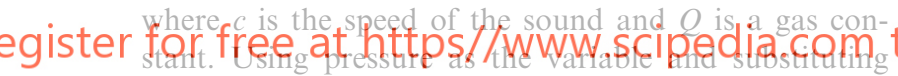
Eqs. (37.1), (37.2) and (37.3) in the LHS of Eq. (25) one obtains the following values for $\alpha$ in the discrete equation, Eq. (32):

$\alpha=0$ for incompressible flows,

$\alpha=\frac{1}{c^{2}}$ for slightly compressible flows,

$\alpha=\frac{1}{Q T^{n+1}} \quad$ for perfect gases.

For high viscosity magmas above the exsolution level and below the fragmentation level, the mixture can be considered as a bubbly flow, characterized by a liquid continuum with dispersed gas bubbles and crystals in thermal and mechanical equilibrium (Papale, 1996). In this bubbly flow regime the gas bubbles and the liquid can be considered to move with the same velocity along the conduit (Sparks, 1978). A suitable state law for the mixture in the bubbly flow regime domain inside the magma chamber and in the lowermost part of the conduit, is (Folch et al., 1998):

$$
\rho=\frac{\rho_{1}}{1+\left(\left(\rho_{1} Q T\right) / P\right)\left(\left(\mathrm{wt}_{\mathrm{o}} \mathrm{q}-s P^{m}\right) /\left(1-s P^{m}\right)\right) \theta\left(P_{\mathrm{c}}-P\right)},
$$

where $\rho$ is the mixture density, $\rho_{1}$ is the liquid density (here assumed to be a constant), $T$ is the temperature, $\mathrm{wt} \%$ is the volatile mass fraction (water for felsic magmas), $s$ and $m$ are solubility constants and $\theta$ is the step function (defined as $\theta=0$ for $P \geq P_{\mathrm{c}}$ and $\theta=1$ for $\left.P \leq P_{\mathrm{c}}\right)$. The critical pressure $P_{\mathrm{c}}$ at which gas exsolution begins depends only on the volatile mass fraction (e.g. Tait et al., 1989):

$P_{\mathrm{c}}=\left(\frac{\mathrm{wt} \%}{s}\right)^{1 / m}$.

Below this critical pressure, where the magma is not vesiculated, we have $\theta=0$ in Eq. (39) and the density is that of the liquid. Fig. 1 shows the mixture density predicted by Eq. (39) for a rhyolitic magma with $\rho_{1}=2400 \mathrm{~kg} / \mathrm{m}^{3}$ at $T=850^{\circ} \mathrm{C}$. Following Tait et al. (1989), values of $s=4.11 \times 10^{-6} \mathrm{~Pa}^{-1 / 2}$ and $m=0.5$ for $\mathrm{H}_{2} \mathrm{O}$ in a rhyolitic melt are assumed. Note how the mixture density is constant below the exsolution level and decreases progressively above this level due to vol-

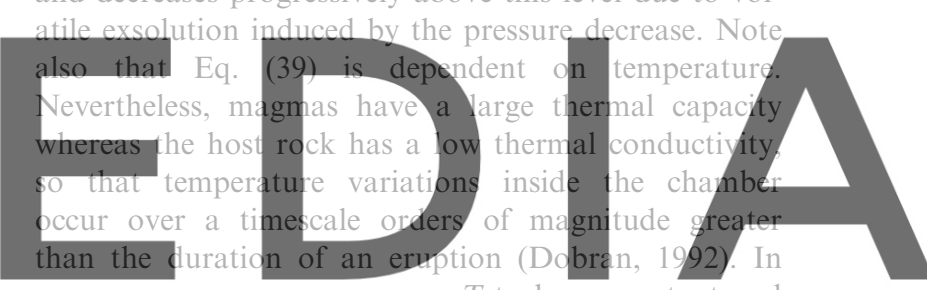

consequence, one may assume $T$ to be a constant and one can assume that Eq. (39) conforms to a barotropic

downd@achtheuversion waithoubathe watermark obtained approximately by multiplying Eq. (39) by $p^{n+1} / p^{n}$, so that:

$$
\alpha=\frac{\rho_{1}}{p^{n}+\rho_{1} Q T\left(\left(\mathrm{wt}_{\%}-s\left(p^{n}\right)^{m}\right) /\left(1-s\left(p^{n}\right)^{m}\right)\right) \theta\left(p_{c}-p^{n}\right)} .
$$

\subsection{General features and discussion}

We have introduced four different parameters $\vartheta_{i} \in[0$, 1] that depending on their values yield fully implicit, semi-implicit or fully explicit solutions to the algorithm (see Table 2). We consider for simplicity $\vartheta_{4}=0$ for the heat equation. The parameter $\vartheta_{1}$ appears in the timediscretized continuity equation and controls both the temporal precision, which is maximum when $\vartheta_{1}=1 / 2$, and the additional stabilizing artificial diffusion, which is maximum when $\vartheta_{1}=1$ and vanishes when $\vartheta_{1}=0$. The parameter $\vartheta_{2}$ is introduced during the splitting of the momentum equation. When zero, the algorithm is fully explicit when $\vartheta_{3}$ is also zero, or semi-implicit if $\vartheta_{3}$ 


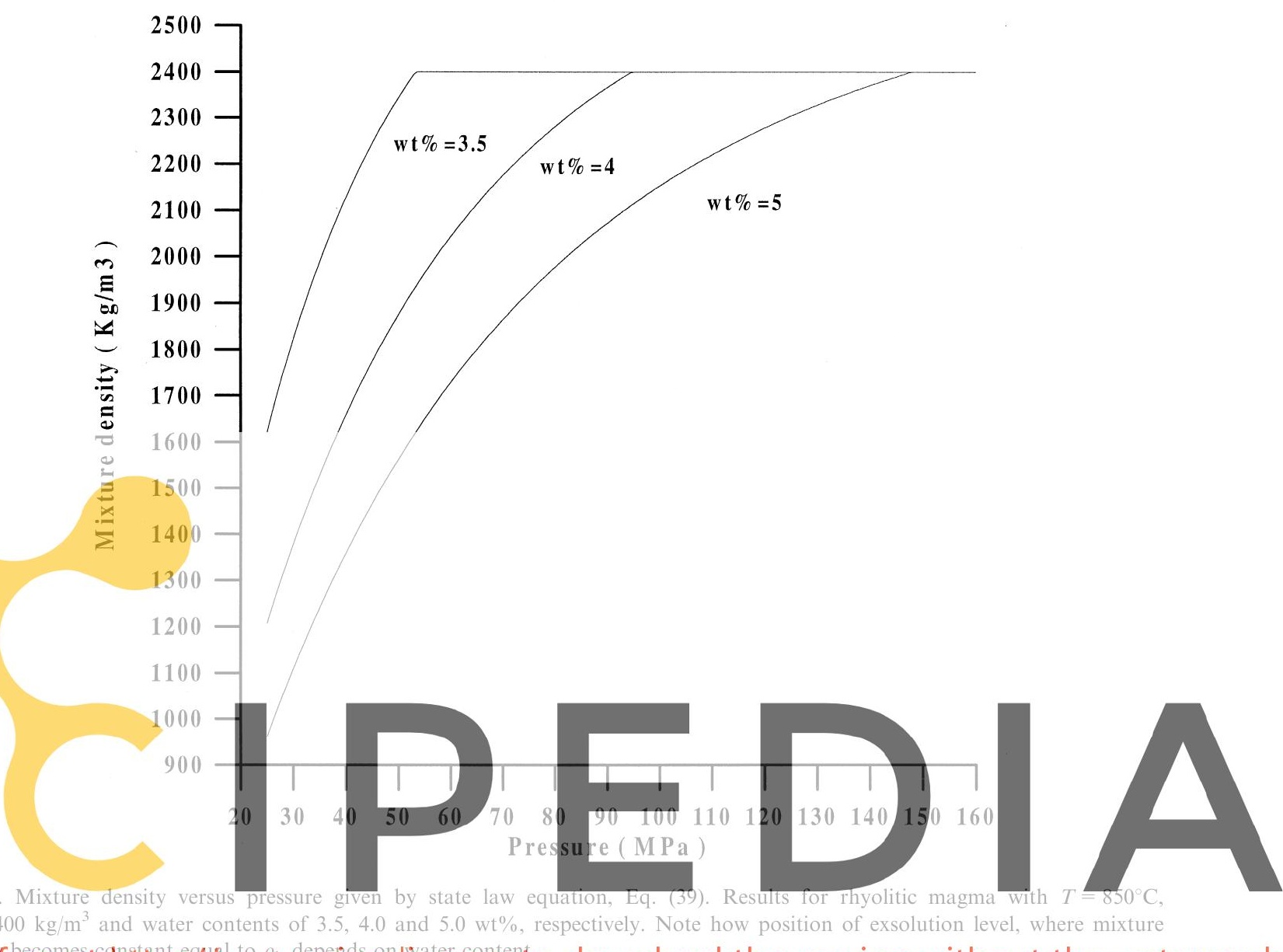

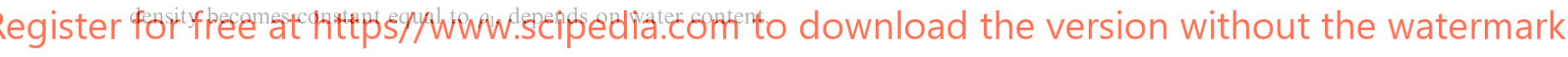

is not zero. If $\vartheta_{2}$ is not zero, the continuity equation is solved implicitly but the algorithm can also be semiimplicit if $\vartheta_{3} \neq 0$. Finally, the parameter $\vartheta_{3}$ appears during the discretization of the momentum equation. It controls whether the viscous and convective terms of this equation are treated explicitly (if $\vartheta_{3}=0$ ) or implicitly (if $\vartheta_{3} \neq 0$ ). To summarise, if $\vartheta_{2}=\vartheta_{3}=0$ the algorithm is fully explicit and if $\vartheta_{2}=0$ and $\vartheta_{3} \neq 0$ it is semi-implicit. Neither the explicit nor the semi-implicit forms of the algorithm deal with the incompressible

Table 2

Characteristics of algorithm depending on values of $\vartheta_{2}$ and $\vartheta_{3}$. We have assumed $\vartheta_{4}=0$ for energy equation (explicit treatment). Parameter $\vartheta_{1}$ can have any value in $[0,1]$

\begin{tabular}{llll}
\hline$\vartheta_{2}$ & $\vartheta_{3}$ & Algorithm & Variables \\
\hline 0 & 0 & fully explicit & $\begin{array}{l}\text { only } \rho \text { can be used as unknown; not possible } \\
\text { for incompressible flows }\end{array}$ \\
$\begin{array}{l}\text { Continuity equation, } \\
\text { Eq. (32), is explicit }\end{array}$ & $0<\vartheta_{3} \leq 1$ & semi-implicit, Eq. (31) is implicit & \\
$0<\vartheta_{2} \leq 1$ & & semi-implicit, Eq. (31) is explicit & $\begin{array}{l}\text { either } p \text { or } \rho \text { can be used for compressible flows, } \\
\text { but only } p \text { for incompressible }\end{array}$ \\
$\begin{array}{l}\text { Continuity equation, } \\
\text { Eq. (32), is implicit }\end{array}$ & $0<\vartheta_{3} \leq 1$ & fully implicit & \\
\hline
\end{tabular}


be said that, from a physical point of view, this model presents some limitations because it assumes a magma with constant viscosity as well as a chemically homogeneous magma composition. Despite these limitations, the model is useful not only to test the algorithm but also to obtain a preliminary approach to this complex physical process. A rhyolitic magma with $\rho_{1}=2400 \mathrm{~kg}$ / $\mathrm{m}^{3}, T=850^{\circ} \mathrm{C}$ and $\mu=10^{5}$ is assumed. The magma chamber is an ellipsoid with a major axis $a=2.5 \mathrm{~km}$, a minor axis $b=0.5 \mathrm{~km}$, a volume $V=4 / 3 \pi a^{2} b \cong 14$ $\mathrm{km}^{3}$ and a conduit radius of $25 \mathrm{~m}$. We utilized a mesh comprising 3080 linear elements (3190 nodes). The initial pressure in the chamber is equal to lithostatic, plus an overpressure $\Delta P=10 \mathrm{MPa}$. This overpressure is typical of the tensile strength of volcanic rocks (Touloukian et al., 1981). The lithostatic pressure at the computational outlet is set to $105 \mathrm{MPa}$. The results show that the overpressure decreases exponentially as the eruption proceeds, until it becomes zero and the eruption stops. This is a general behaviour found in any other simulation (Folch et al., 1998). This pressure decrease produces a drop in the exsolution level, so that deeper parts of the chamber become progressively volatile-oversaturated. Thus, the upper, oversaturated magma layer becomes progressively thicker and less dense. Fig. 4 shows the eruption rate for this particular simulation using water contents of 4.5 and 5.0 in wt $\%$. In all of the numerical experiments, the eruption rate reaches a peak immediately after the onset of the eruption and then decreases exponentially as the eruption proceeds. In this particular example, a total erupted mass of approximately $3 \times 10^{12} \mathrm{~kg}$ is obtained. This value is comparable with those found for explosive volcanic eruptions: $0.6 \times 10^{12} \mathrm{~kg}$ for the 1980 Mount St. Helens eruption (Scandone and Malone, 1985), 5$73 \times 10^{12} \mathrm{~kg}$ for the Nisyros eruption (Barberi et al., 1988 ) and $8.6 \times 10^{12} \mathrm{~kg}$ for the 79 A.D. Vesuvius eruption (Barberi et al., 1981). Fig. 5 shows the position of the exsolution level versus time for water contents of 4.3 and $4.5 \mathrm{wt} \%$. For $\mathrm{wt} \%=4.3$ the exsolution level, which is initially located $275 \mathrm{~m}$ below the conduit entrance, drops approximately $300 \mathrm{~m}$ in 22 h. Fig. 6 shows the velocity field at the conduit entrance after $22 \mathrm{~h}$ of eruption. A commonplace feature found during
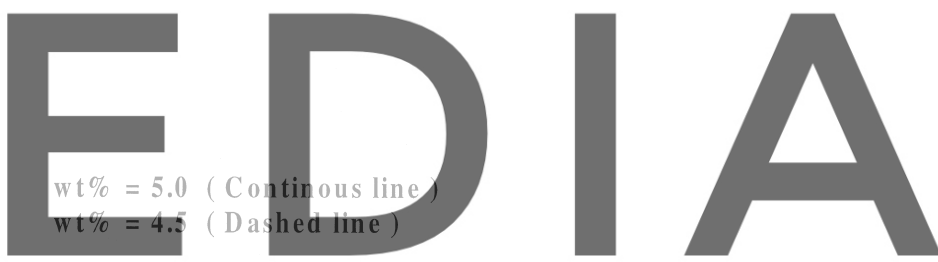

legister for free at https/晌WHW.scipedia.com to download the version without the watermark

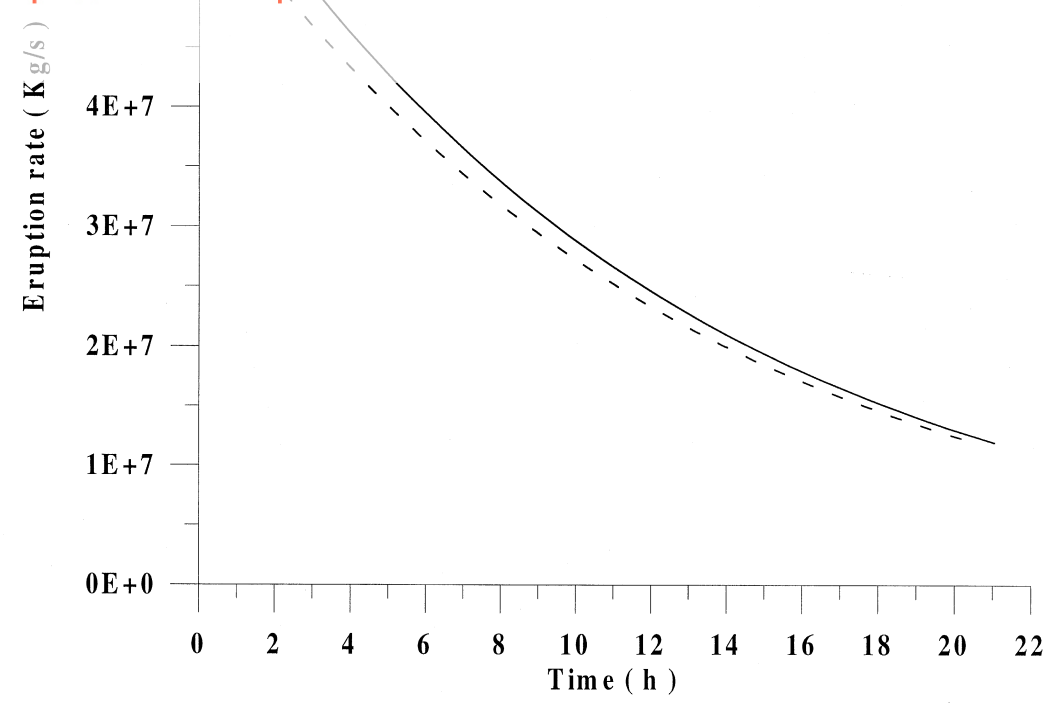

Fig. 4. Eruption rate versus time. Results for initial overpressure $\Delta P=10 \mathrm{MPa}$, viscosity $\mu=10^{5} \mathrm{~Pa} \mathrm{~s}$, liquid density $\rho_{\mathrm{l}}=2400 \mathrm{~kg} /$ $\mathrm{m}^{3}$ and water contents of 4.5 and $5.0 \mathrm{wt} \%$. Exponential decrease in mass discharge rate is only slightly dependent on volatile content. 


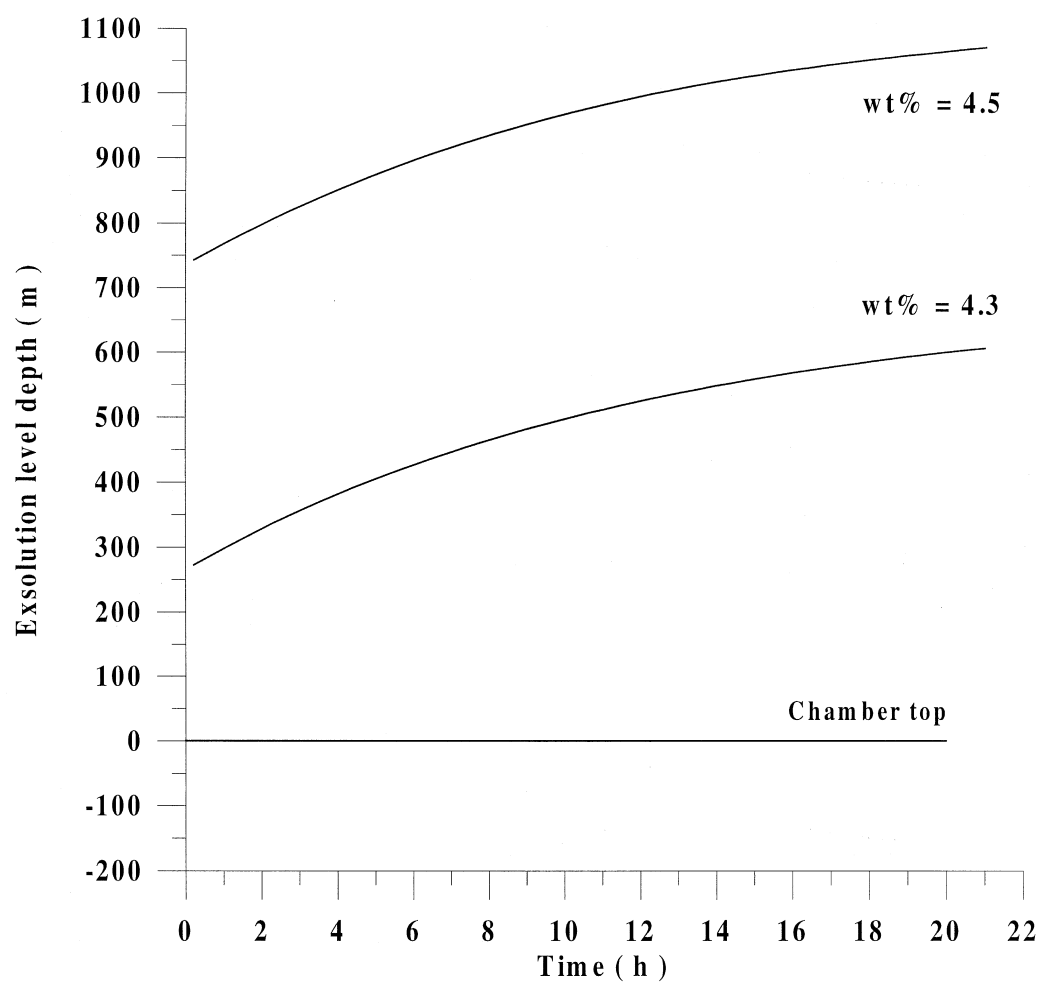

Fig. 5. Position of exsolution level. Origin of vertical axis set at top of chamber. Same parameters as in Fig. 4, but with water contents of 4.3 and $4.5 \mathrm{wt} \%$. Note that shape of functions does not differ substantially when water content is changed. However, increasing volatile contents produces movement of exsolution level.

the withdrawal of magma from chemically homogeneous chambers is that the magma reaches the conduit entrance laterally. Note that although the velocity field is unsteady, its changes are given in a characteristic time of hours, so that at any instant we can approach the streamlines by the trajectories. A volume of magma reaching the conduit entrance is partly erupted through the conduit and partly reinjected back into the chamber. Thus, a volume of magma initially emplaced at the top of the chamber moves first laterally to the vicinity of the conduit entrance and is then either erupted or forced to descend hundreds of meters back into the chamber before again moving up. As this magma descends, the exsolved volatiles within it are progressively re-dissolved back into the magma as the pressure raises increases their solubility in the melt. This phenomenon has an important consequence: as we have assumed that the magma behaves as an incompressible flow below the exsolution level, any inflow into the undersaturated incompressible layer must produce an equal outflow to ensure the conservation of mass. This movement is transmitted through viscous stresses, so that eventually, the entire magma chamber is affected by this induced movement.

\section{Summary and discussion}

We have developed an algorithm that numerically solves the Navier-Stokes equations using a finite-element method. The algorithm uses a fractional-step method which allows the use of equal interpolation spaces for the pressure and velocity fields and produces a stabilizing effect on the pressure, eliminating the need for a special interpolation at the incompressible limit. This algorithm can be used to model many problems in geological fluid dynamics. We apply it to characterize the withdrawal of shallow magma chambers during explosive volcanic eruptions. The results show that the pressure at the conduit entrance decreases exponentially as the eruption proceeds. This decrease in pressure produces a drop in the exsolution level, so that deeper parts of the chamber become progressively volatile-oversaturated with time. The total 

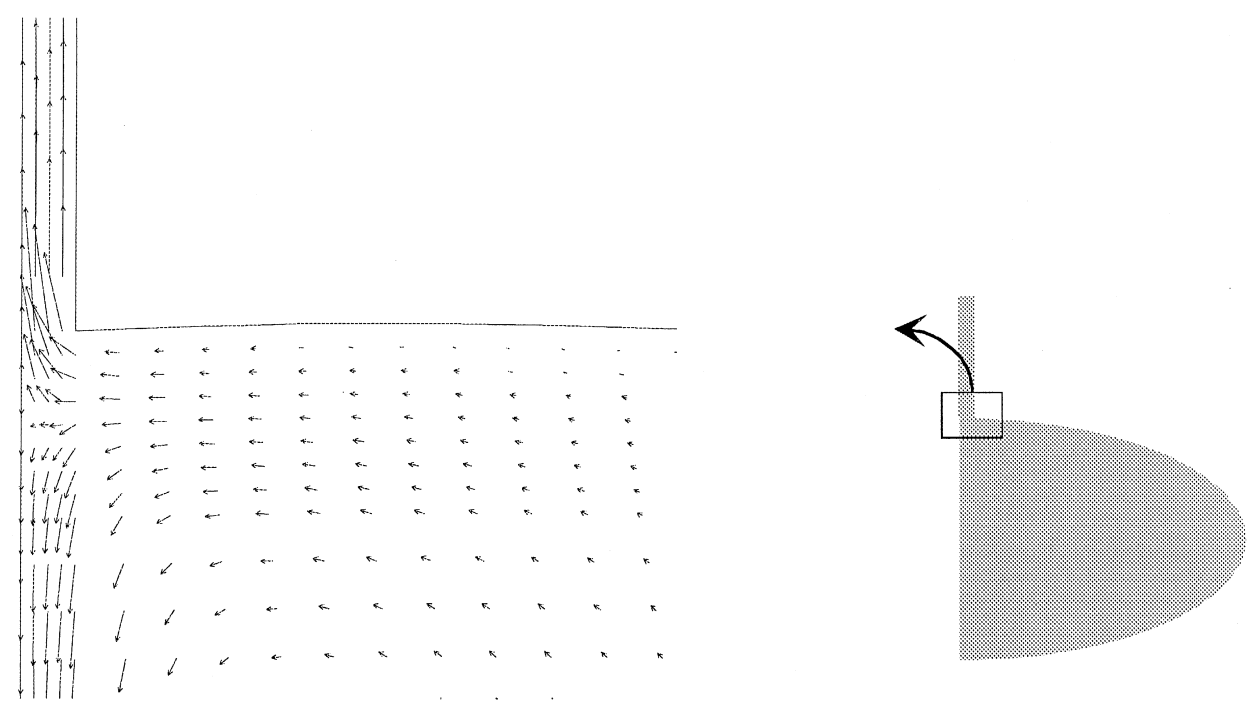

Fig. 6. Velocity field at conduit entrance after $22 \mathrm{~h}$ of simulated eruption using the same chamber geometry and magma properties as for Figs. 4 and 5. Although velocity field is unsteady, its temporal variations are slow and one can approach streamlines by trajectories. Note how a volume of magma reaches conduit entrance laterally and is then either erupted or reinjected back into the chamber.

mass erupted is in good agreement with the results of Bower and Woods (1997), who found that for volatilesaturated magma chambers a mass fraction of $0.01-0.1$ of the initial mass will be erupted. In general, we deduce that for chambers with similar volume and magma properties, the qualitative behavior does not change substantially when different chamber geometries are considered. However, the quantitative behavior does vary slightly. The total erupted mass increases as the chamber becomes flatter, because flattened chambers have a large volumetric fraction of compressible oversaturated magma. A small change in the initial volatile content does not change the mass discharge rate substantially, but has an important influence on the position of the exsolution level. A small increase in volatile content produces a substantial drop in the exsolution level, which reaches deeper parts of the chamber. The total erupted mass does not depend on the viscosity, although the viscosity plays a major role in controlling the duration and the peak intensity of the eruption. An increase in the initial overpressure, associated in the model with the tensile strength of the host rock, produces an increase in the total downward displacement of the exsolution level. In consequence, when the initial overpressure is high, a greater mass is erupted because a greater volumetric fraction of magma changes from volatile-undersaturated and incompressible to volatile-oversaturated and compressible during the withdrawal process.

\section{Acknowledgements}

This research has been founded by the EC contract ENV-CT96-0259 and the CICYT Project AMB960498-C04. A.F. is grateful for a CIRIT research fellowship. We also thank Professor Yuen, an anonymous reviewer and Giray Ablay for their comments on this paper.

\section{References}

Barberi, F., Bizourard, H., Clocchiatti, R., Metrich, N., Santacroce, R., Sbrana, A., 1981. The Somma-Vesuvius magma chamber: a petrological and volcanological approach. Bulletin of Volcanology 44, 295-315.

Barberi, F., Navarro, J., Rosi, M., Santacroce, R., Sbrana, A., 1988. Explosive interactions of magma with ground water: insights from xenoliths and geothermal drillings. Rendo. Soc. Itl. Mineral. Petrol. 43, 901-926.

Batchelor, G., 1967. An Introduction to Fluid Dynamics, 1st ed. Cambridge University Press, Cambridge, 615 pp.

Blake, S., 1981. Volcanism and dynamics of open magma chambers. Nature 289, 83-785.

Blake, S., 1984. Volatile oversaturation during the evolution of silicic magma chambers as an eruption trigger. Journal of Geophysical Research 89 (B10), 8237-8244.

Bower, S., Woods, A., 1997. Control of magma volatile content and chamber depth on the mass erupted during explo- 
sive volcanic eruptions. Journal of Geophysical Research 102 (B5), 10273-10290.

Chorin, A.J., 1967. A numerical method for solving incompressible viscous problems. Journal Computational Physics 2, 12-26.

Chorin, A. J., 1969. On the convergence of discrete approximation to the Navier-Stokes equations. Mathematical Computation 23, 745-762.

Codina, R., Vázquez, M., Zienkiewicz, O., 1998. A general algorithm for compressible and incompressible flow. Part III. The semi-implicit form. International Journal for Numerical Methods in Fluids 27, 13-32.

Dobran, F., 1992. Nonequilibrium flow in volcanic conduits and application to the eruptions of Mt. St. Helens on May 18, 1980, and Vesuvius in AD 79. Journal of Volcanology and Geothermal Research 49, 85-311.

Faber, T., 1995. Fluid Dynamics for Physicists. Cambridge University Press, Cambridge, $440 \mathrm{pp}$.

Folch, A., Martí, J., Codina, R., Vázquez, M., 1998. A numerical model for temporal variations during explosive central vent eruptions. Journal of Geophysical Research 103 (B9), 20883-20899.

Hirsch, C., 1990. Numerical Computation of Internal and External Flows. John Wiley \& Sons, New York, 740 pp.

Papale, P., 1996. Modeling of magma ascent along volcanic conduits: a review. Environment and Climate Programme. European Commission, Brussels, pp. 3-40.

Papale, P., Dobran, F., 1993. Modeling of the ascent of magma during the plinian eruption of Vesuvius in AD 79. Journal of Volcanology and Geothermal Research 58, 101132.

Papale, P., Dobran, F., 1994. Magma flow along the volcanic conduit during the Plinian and pyroclastic flow phases of the May 18, 1980, Mount St. Helens eruption. Journal of Geophysical Research 99, 4355-4373.

Scandone, R., Malone, S., 1985. Magma supply, magma discharge and readjustment of the feeding system of the
Mount St. Helens during 1980. Journal of Volcanology and Geothermal Research 23, 239-262.

Simo, J., Armero, F., 1994. Unconditional stability and long behavior of transient algorithms for the incompressible Navier-Stokes equations. Computational Methods in Applied Mechanics and Engineering 111, 111-154.

Smith, R.L., 1979. Ash flow magmatism. American Geological Society 180, 5-27.

Sparks, R.S.J., 1978. The dynamics of bubble formation and growth in magmas: a review and analysis. Journal of Volcanology and Geothermal Research 3, 1-37.

Spera, F., 1984. Some numerical experiments on the withdrawal of magma from crustal reservoirs. Journal of Geophysical Research 89 (B10), 8222-8238.

Spera, F., Yuen, D., Greer, J., Sewell, G., 1986. Dynamics of magma withdrawal from stratified magma chambers. Geology 14, 723-726.

Tait, S., Jaupart, C., Vergniolle, S., 1989. Pressure, gas content and eruption periodicity of a shallow, crystallizing magma chamber. Earth and Planetary Science Letters 92, $107-123$.

Touloukian, S., Judd, R., Roy, R., 1981. Physical Properties of Rocks and Minerals. McGraw-Hill, New York, 548 pp.

Trial, F., Spera, F., Greer, J., Yuen, D., 1992. Simulations of magma withdrawal from compositionally zoned bodies. Journal of Geophysical Research 97 (B5), 6713-6733.

Vázquez, M., Codina, R., Zienkiewicz, O., 1996. A fractional step method for the solution of the Navier-Stokes equations. Internal Report. CIMNE, Barcelona, 103 pp.

Zienkiewicz, O., Codina, R., 1995. A general algorithm for compressible and incompressible flow. Part I. The split, characteristic-based scheme. International Journal for Numerical Methods in Fluids 20, 869-885.

Zienkiewicz, O., Morgan, K., Satya, B., Codina, R., Vázquez, M., 1995. A general algorithm for compressible and incompressible flow. Part II. Tests on the explicit form. International Journal for Numerical Methods in Fluids 20, 887-913. 